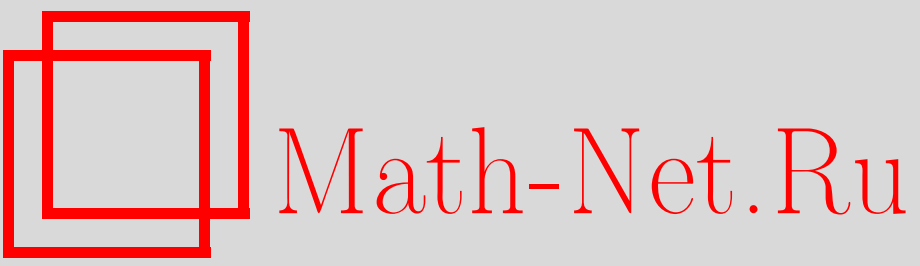

А. Г. Дюбина, Пример скорости ухода на бесконечность для случайного блуждания по группе, УМH, 1999, том 54, выпуск 5, 159-160

DOI: https://doi.org/10.4213/rm208

Использование Общероссийского математического портала Math-Net.Ru подразумевает, что вы прочитали и согласны с пользовательским соглашением

http://www.mathnet.ru/rus/agreement

Параметры загрузки:

IP: 54.224 .187 .69

26 апреля 2023 г., 18:09:41 


\title{
ПРИМЕР СКОРОСТИ УХОДА НА БЕСКОНЕЧНОСТЬ ДЛЯ СЛУЧАЙНОГО БЛУЖДАНИЯ ПО ГРУППЕ
}

\author{
А. Г. ДюбинА
}

Как растет средняя длина слова при случайном блуждании по группе? Часто порядок роста линейньй, в частности, это так для любой неаменабельной группы. Если же рост более медленный, чем линейный, во многих примерах он асимптотически равен $\sqrt{n}$ - например, в любой абелевой группе. Группа афффинных преобразований диадической прямой имеет экспоненциальный рост, но средняя длина тоже растет как $\sqrt{n}$. Оказывается, что, тем не менее, есть и другие возможности для среднего роста:

Теорема 1. В сплетении $\mathbb{Z}^{2} \imath \mathbb{Z} / 2 \mathbb{Z}$ существуют такие образующие, что для определяемого ими простого случайного блуждания

$$
\mathrm{E}_{\mu * n}[l] \asymp \frac{n}{\ln n} .
$$

Здесь $l$-длина в словарной метрике, а $\mu$-мера, равномерно распределенная на въбранных образующих и обратных $к$ ним.

Для доказательства теоремы 1 достаточно доказать две леммы.

ОПРЕДЕЛЕНИЕ. Назовем размахом случайного блуждания количество различных элементов группы, посещенных за первые $n$ шагов $(n \geqslant 0)$. Обозначим размах через $R_{n}$.

Лемма 1. Рассмотрим простое симметричное случайное блуждание на $\mathbb{Z}^{2}$. Тогда

$$
\mathrm{E}\left[R_{n}\right] \sim \frac{\pi n}{\ln n} .
$$

ДокАЗАТЕЛЬСтво. Пусть $F_{n}(0,0)$ - это вероятность того, что в момент $n$ блуждание впервые возвращается в 0 . Пусть $\varphi_{k}$ - случайная величина, принимающая значение 1 , если на $k$-м шаге блуждание попадает в ранее не посещенную точку, и 0 иначе. Тогда

$$
\mathrm{E}\left[\varphi_{k}\right]=1-\sum_{j=1}^{k} F_{j}(0,0)
$$

$[2 ; \S 4$, доказательство теоремы 1$]$. Пусть $T$ - время первого возврашения в 0 . Тогда

$$
1-\sum_{j=1}^{k} F_{j}(0,0)=\operatorname{Pr}[T>k] .
$$

Но для простого двумерного случайного блуждания

$$
\operatorname{Pr}[T>n] \sim \frac{\pi}{\ln n}
$$

$[2 ; \S 16$, пример 1]. Поэтому

$$
\mathrm{E}\left[\varphi_{k}\right] \sim \frac{\pi}{\ln k}
$$

Тогда

$$
\mathrm{E}\left[R_{n}\right]=\sum_{i=1}^{n} \mathrm{E}\left[\varphi_{k}\right] \sim \sum_{i=1}^{n} \frac{\pi}{\ln i} \sim \frac{\pi n}{\ln n}
$$


Лемма 2. Пусть $A$ - конечно-порожденная группа, пусть $a_{1}, \ldots, a_{k}-$ ее образующе, а $\delta$ - это образующая $\mathbb{Z} / 2 \mathbb{Z}$. Рассмотрим на $B=A \imath \mathbb{Z} / 2 \mathbb{Z}$ меру, равномерно распределенную на образующ, их

$$
\left(a_{i}, e\right),\left(a_{i}, \chi(\delta)\right),(e, \chi(\delta))\left(a_{i}, e\right),(e, \chi(\delta))\left(a_{i}, e\right)(e, \chi(\delta))
$$

(әде $\chi(\delta)$ - функиия из $А$ в В такая, что $\chi(\delta)(e)=\delta, \chi(\delta)(x)=$ е при $x \neq e$ ). Тогда

$$
\frac{1}{4} \mathrm{E}\left[R_{n}^{A}\right] \leqslant \mathrm{E}_{\mu^{* n}}[l] \leqslant 2 \mathrm{E}\left[R_{n}^{A}\right] .
$$

ДокАЗАТЕЛЬСтво. Второе неравенство следует из того, что

$$
l_{B}(g) \leqslant 2 R_{n}^{A}
$$

где $g$ - элемент, посешенный на $n$-м шаге блуждания, а $R_{n}^{A}$ - размах проекции траектории на $A$.

Для доказательства первого неравенства подсчитаем условное математическое ожидание

$$
\mathrm{E}_{\mu^{* n}}\left[l \mid R_{n}^{A}=R\right]
$$

Пусть $g=(a, f)(a \in A, f: A \rightarrow \mathbb{Z} / 2 \mathbb{Z})$. Пусть $S_{n}$ - это количество элементов в носителе $f$. Тогда $l_{B}(g) \geqslant \frac{1}{2} S_{n}$, так как умножение на любой из рассматриваемых образующих изменяет значение функции не более чем в двух точках. Заметим, что

$$
\mathrm{E}\left[S_{n}^{B} \mid R_{n}^{A}=R\right]=\frac{1}{2} R .
$$

Действительно, заметим, что в каждом из $R$ посещенных элементов группы $A$ значение функции $f$ с вероятностью $\frac{1}{2}$ равно $\delta$. Поэтому

$$
\mathrm{E}_{\mu^{* n}}\left[l \mid R_{n}^{A}=R\right] \leqslant \frac{1}{4} R
$$

откуда следует первое неравенство леммы. Приведем теперь без доказательства серию примеров, реализующих степенные асимптотики.

Теорема 2. Рассмотрим группь, определяемые рекуррентно:

$$
G_{1}=\mathbb{Z} ; \quad G_{i+1}=\mathbb{Z} \imath G_{i} .
$$

В $G_{k}$ существуют такие образующие, что для определяемого ими простого случайного блуждания

$$
\mathrm{E}_{\mu^{* n}}[l] \asymp n^{\frac{2^{k}-1}{2^{k}}} .
$$

Автор выражает благодарность А. М. Вершику за постановку задачи и полезные обсуждения.

\section{СПИСОК ЛИТЕРАТУРЫ}

[1] Вершик А. М. // Зап. научн. семин. ЛОМИ. 1999. Т. 256. С. 5-18. [2] Spitzer F. Principles of Random Walk. Princeton: Van Nostrand, 1964.

Принято редколлегией 23.08.1999 